\title{
O Ouvido Absoluto: bases neurocognitivas e perspectivas
}

\author{
Fabrízio Veloso - Universidade de Brasilia, Brasilia/DF, Brasil \\ Maria Angela Guimarães Feitosa - Universidade de Brasilia, Brasilia/DF, Brasil
}

\begin{abstract}
Resumo
O Ouvido Absoluto, a capacidade de nomear tons sem uma referência externa, é comumente relacionada à cognição musical. Embora se note um crescente investimento em seu estudo, muitas questões ainda encontram-se pouco esclarecidas, tais quais: gênese, conceito, metodologias de estudo, aspectos estruturais e funcionais que o caracterizam. Esta revisão propõe-se a analisar os avanços recentes e limitações da área, considerando-se as bases metodológicas e teóricas, bem como pontos controversos que permeiam o tema, como a definição e origem da habilidade. Como conclusão, aponta-se a necessidade de delineamentos específicos para o estudo da cognição musical como um aliado importante para a melhor compreensão e elucidação dos problemas inerentes ao Ouvido Absoluto.

Palavras-chave: Ouvido absoluto; Cognição musical; Percepção musical; Neurociência cognitiva; Percepção auditiva.
\end{abstract}

\section{Absolute Pitch: neurocognitive foundations and perspectives}

\begin{abstract}
Absolute pitch, the ability to name tones without an external reference, is commonly related to music cognition. Despite the growing investment in its understanding, many questions are still poorly understood, such as genesis, conceptualization, research methodology, identification of structural and functional features. The present review aims to analyze recent advances and limitations in the area, considering its methodological and theoretical bases, as well as existing controversies (definition and genesis). It concludes by pointing the need for specific designs for the study of musical cognition for a better understanding of absolute pitch.

Keywords: Absolute pitch; Music cognition; Music perception; Cognitive neuroscience; Auditory perception.
\end{abstract}

El Oído Absoluto: bases neurocognitivas y perspectivas

\begin{abstract}
Resumen
El Oído Absoluto, la capacidad de nombrar tonos sin una referencia externa, está comúnmente relacionado con la cognición musical. Aunque se note una creciente inversión en su estudio, muchas cuestiones aún se encuentran poco aclaradas, tales como: génesis, concepto, metodologías de estudio, aspectos estructurales y funcionales que lo caracterizan. Esa revisión propone analizar los avanzos y limitaciones del área, considerando las bases metodológicas y teóricas y puntos controvertidos que tratan el tema, como la definición y origen de la habilidad. Por conclusión, se demuestra la necesidad de delineaciones específicas para el estudio de la cognición musical como un importante aliado para una mejor comprensión y aclaración de los problemas inherentes al Oído Absoluto.

Palabras clave: Oído absoluto; Cognición musical; Percepción musical; Neurociencia cognitiva; Percepción auditiva.
\end{abstract}

A literatura descreve o Ouvido Absoluto como a capacidade de identificar, nomear ou produzir a frequência de um estímulo tonal sem o auxílio de um tom de referência (Deutsch, 2002; Ross, Olson \& Gore, 2003; Ward, 1999). Associa-se a ele uma capacidade diferenciada na codificação do croma, ou seja, a repetição cíclica de intervalos em cada oitava (Roederer, 2002) e da altura tonal de um tom musical (Bachem, 1937).

Sua ocorrência é rara, com prevalência estimada em 1 para cada 10.000 pessoas, considerando-se a população da Europa e América do Norte, e é mais comumente observada em músicos (cerca de 20\%) com treinamento formal (Bachem, 1955; Profita \& Bidder, 1988). Isso se deve, basicamente, a dois fatores: a assunção recorrente de que o ouvido absoluto se limita à capacidade de nomeação de uma nota musical (o que se reflete na elaboração de testes que ratifiquem a existência dessa capacidade) e no fato de que, invariavelmente, não-músicos não são hábeis em reconhecer essa capacidade. Os primeiros estudos sobre o ouvido absoluto contemplavam diferenças fundamentais na definição do termo. Bachem (1937) chamava a atenção para subtipos: ouvido absoluto genuíno, quase ouvido absoluto e pseudo-ouvido absoluto, sendo que pessoas com quase ouvido absoluto se difeririam pela interiorização de apenas uma frequência específica que sirva como referência para o reconhecimento dos tons, como um Lá4 (440 $\mathrm{Hz}$ ), ao passo que pessoas com pseudo-ouvido Absoluto seriam capazes apenas de fazer uma estimativa acerca da frequência tocada, em detrimento da codificação tonal exata, com base em treinamento musical prévio.

O objetivo desta revisão é identificar e discutir os tópicos controversos acerca do ouvido absoluto, analisando a literatura científica atual relacionada ao tema e incluindo os trabalhos clássicos. Vale ressaltar a relevância científica do assunto, considerando-se que a explanação da habilidade traz implicações importantes acerca do processamento musical e dos aspectos perceptuais, cognitivos, bem como os correlatos neurais envolvidos na resolução de informações sonoras de alta complexidade e como estas afetam a dinâmica cerebral. Os artigos examinados foram pesquisados na base de dados "Web of Science" e "Scopus", usando as palavras-chave "absolute pitch", "music", "music perception" e "music cognition". 
Foram considerados, basicamente, textos publicados em língua inglesa, sem a delimitação do período de publicação. Consideraram-se os artigos disponibilizados na base de dados entre o período de agosto de 2010 e junho de 2012. As referências citadas pelos artigos centrais ao tema e obtidos na base de dados também foram utilizadas para identificação de outros estudos relevantes.

\section{Percep̧̧ão e cognição}

Pesquisas nas duas últimas décadas consideram que o ouvido absoluto se relaciona à capacidade de associar automaticamente uma frequência tonal a um rótulo linguístico que a identifique enquanto uma nota musical (Levitin \& Rogers, 2005; Schulze, Mueller \& Koelsch, 2012), bem como a manutenção dessa informação em um sistema de memória de longo prazo (Levitin, 1994). Nota-se que, comumente, o fenômeno se refere ao conhecimento prévio das notas musicais, em acordo com a escala temperada. Em outras palavras, define-se o ouvido absoluto ao se verificar elevado grau de precisão na associação direta entre um estímulo sonoro e um rótulo verbal específico. Portanto, nota-se participação evidente de um componente linguístico (cognitivo) relacionado à habilidade que o diferencia das demais manifestações da música no cérebro humano, além do conhecimento prévio em teoria musical como condição intrínseca.

O papel crucial da cognição na habilidade é notório objeto de atenção na literatura. Estudos evidenciam um desempenho marcadamente diferenciado desta população no que se refere à acurácia para nomeação de notas musicais, quando comparadas com músicos sem a habilidade ou pessoas sem treinamento musical (Baharloo \& cols., 1998; Takeushi \& Hulse, 1993;) e à velocidade da identificação de tons musicais (Miyazaki, 1988). Ainda, as estruturas ativadas no cérebro para tarefas de nomeação e identificação de determinados tons musicais em pessoas com ouvido absoluto se diferenciam das áreas ativadas em pessoas que não possuem essa habilidade (Schlaug, Jancke, Huang \& Steinmetz, 2005; Wilson, Lusher, Wan, Dudgeon \& Reutens, 2009; Zatorre, Perry, Beckett, Westbury \& Evans, 1998). Em conjunto, esses achados corroboram o papel distinto e pontual da cognição na identificação e produção de um tom musical em possuidores de ouvido absoluto.

$\mathrm{O}$ ouvido absoluto também tem sido associado à codificação de frequências de sinais acústicos distintos de estímulos musicais (Ross, Gore \& Marks, 2005). Alguns estudos consideram o componente perceptual como sendo essencial ao fenômeno, em contraponto a uma abordagem essencialmente cognitiva. Em pesquisas anteriores de Ross e cols. (2004), verificou-se que em tarefa de ajuste de frequência tonal a uma frequência previamente apresentada, tanto músicos sem treinamento musical formal podem apresentar desempenho equivalente ao de músicos com ouvido absoluto (Ross, Olson, Marks \& Gore, 2004), quanto adultos não-músicos parecem ser capazes de codificar adequadamente a frequência de estímulos musicais em tarefa de identificação de frequência tonal (Ross \& cols., 2003). Além disso, pessoas sem ouvido absoluto também possuem a capacidade de identificar mudanças no nível de altura dos tons que compõem trechos de músicas conhecidas por eles (Deutsch, 2002; Halpern, 1989; Schellenberg \& Trehub, 2003), sugerindo a retenção da informação acústica. Da mesma forma, músicos sem ouvido absoluto podem reter uma informação tonal na memória de longo prazo em tarefa de reconhecimento de melodias (Creel \& Tumlin, 2012) e julgamento de frequência correta do tom de telefone (Smith \& Schmukler, 2004), bem como na identificação da tonalidade correta em músicas conhecidas (Terhardt \& Seewann, 1983).

Em conjunto, os estudos acima mencionados dão sustentação à assertiva de que a exclusão de pessoas sem treinamento musical ou sem ouvido absoluto para o estudo do tema pode traduzir, portanto, uma limitação importante para a melhor compreensão do fenômeno. Ainda, esses estudos apontam que tanto componentes cognitivos quanto perceptuais exercem papel importante no desenvolvimento do ouvido absoluto. Considerá-las isoladamente pode implicar uma limitação crucial para seu entendimento. Os limites de participação de cada um desses fatores, bem como suas interações ainda não são bem estabelecidos pelos estudos e merecem maior investimento científico, considerando-se tanto músicos com a habilidade, bem como potenciais possuidores sem conhecimento teórico musical.

\section{Fatores constitucionais e ambientais}

A gênese da habilidade é outro ponto controverso. Em primeiro lugar, algumas pesquisas vêm demonstrando a influência do treinamento musical formal, considerando-se a existência de um período sensível para o seu desenvolvimento (Miyazaki, 1988; Takeushi \& Hulse, 1993), associado ao desenvolvimento da linguagem (Deutsch, 2002). Parece haver convergência entre os estudos ao se verificar que pessoas que iniciaram estudos musicais em idade inferior a seis anos têm maior propensão a desenvolver ouvido absoluto do que aquelas que iniciaram os estudos em período posterior de desenvolvimento. Em estudo conduzido por Baharloo \& cols. (1998), com músicos de orquestra, conservatórios e programas de treinamento musical, verificou-se que cerca de $40 \%$ possuíam ouvido absoluto e iniciaram seus estudos antes dos 4 anos de idade. Em contrapartida, apenas $4 \%$ dos que iniciaram treinamento musical após 9 anos de idade, relatavam ser possuidores de Ouvido Absoluto. Outro estudo (Deutsch, Dooley, Henthorn 
\& Head, 2009) observou que pessoas com início do treinamento musical formal entre 2 e 5 anos obtiveram maior percentual de respostas corretas em teste de nomeação de notas musicais, sugerindo a influência do treino precoce na prevalência da habilidade. Efetivamente, sugere-se que o período de 2 a 5 anos de idade facilitaria a associação existente entre a representação absoluta de uma frequência tonal e a rotulação verbal desta frequência no sistema de memória de longo prazo (Levitin \& Rogers, 2005; Zatorre \& Beckett, 1989). Em conjunto, estes achados reforçam a influência ambiental na aquisição do ouvido absoluto.

Entretanto, uma explicação constitucional também tem sido adotada. Há evidências de maior prevalência de ouvido absoluto em populações asiáticas, como a japonesa, a coreana e a chinesa (Gregersen, Kowalski, Kohn \& Marvin, 2000), independentemente do início precoce em estudo musical e de serem países com linguagens tonais, bem como taxa elevada de concordância para a ocorrência da habilidade em gêmeos monozigóticos (78\%), em comparação a gêmeos dizigóticos (45\%) (Theusch \& Gitschier, 2011). Outro dado aponta uma prevalência quatro vezes maior desta habilidade em famílias de entrevistados com ouvido absoluto, em comparação com pessoas sem ouvido absoluto (Baharloo \& cols., 1998). Ainda, Baharloo e cols. (1998) verificaram em seus estudos que a maioria dos participantes pesquisados e que relataram treinamento musical formal iniciado com 6 anos ou menos não possuíam ouvido absoluto, sugerindo, portanto, que o treinamento musical precoce não consegue explicar, por si, a ocorrência da habilidade. Adicionalmente, sugeriu-se a influência de componente genético ao se verificar que os participantes com ouvido absoluto relataram a ocorrência de outros possuidores da habilidade em sua família em uma proporção quatro vezes maior do que aqueles sem ouvido absoluto.

Há, ainda, uma explanação integrativa sobre este tópico. De acordo com esta abordagem, evidencia-se uma confluência de fatores estruturais, funcionais, cognitivos e desenvolvimentais do cérebro humano que favorecem o aparecimento da habilidade (Zatorre, 2003). Wilson, Lusher, Martin, Rayner \& McLachlan (2012) destacam a interseção entre histórico familiar e o início do treinamento formal em um sistema (dó fixo) em que se designa uma sílaba (nome da nota) ao tom tocado, como prevalentes entre os participantes com ouvido absoluto. A natureza integrativa dos fatores genético e ambiental da habilidade também se reflete nos padrões específicos da atividade neuronal, tais quais a assimetria estrutural para o processamento auditivo e características morfológicas do cérebro de pessoas com essa habilidade. A ativação mais proeminente de regiões frontais (dorsolateral posterior) em músicos com ouvido absoluto em tarefa de escuta de tons musicais, quando comparada com músicos sem ouvido absoluto (Zatorre, Perry, Beckett, Westbury \& Evans, 1998) é tratada como evidência para a participação de um tipo de memória específica nessa população. Em outros estudos, verificou-se acentuada assimetria do planum temporal esquerdo (Schlaug \& cols., 2005), hiperconectividade de estruturas do lobo temporal bilateral (Loui, Li, Hohmann \& Schlaug, 2011), bem como dominância hemisférica cerebral para identificação de altura (Brancucci, di Nuzzo \& Tommasi, 2009). Ainda, pesquisas demonstraram ativação da parte posterior do giro temporal medial e baixa ativação da parte mesial do giro temporal superior de músicos com ouvido absoluto expostos a estímulos da fala com variação léxica ou prosódica, quando comparados com músicos sem ouvido absoluto (Oechslin \& cols., 2009), sugerindo ativação de vias neurais distintas para o processamento de informações musicais e linguísticas (da fala).

As investigações neurobiológicas acerca do tema são relativamente recentes (Ross, Gore \& Marks, 2005) e vinculam-se, comumente, ao estudo da cognição musical, em uma perspectiva de neurociência cognitiva. Embora a cognição seja uma área clássica de estudo científico na psicologia (Levitin \& Tirovolas, 2009), a aplicação das metodologias desenvolvidas nessa área ao objeto da música ainda é relativamente recente e merecedora de análise cuidadosa, uma vez que o estímulo musical é processado de maneira particular no cérebro humano. Baird e Samson (2009) observam que, no estudo da memória musical, por exemplo, os estudos utilizam protocolos que advêm das metodologias aplicadas ao estudo da linguagem nãomusical. Deve-se ressaltar que um dos principais motivos dessa realidade é o fato de que, por muito tempo, elegeu-se como foco principal dos estudos acerca do cérebro humano, a linguagem formal, comumente associada ao hemisfério dominante (Kandel, Schwartz \& Jessel, 1995), enquanto os trabalhos pioneiros descreviam a atividade musical como uma ocorrência relacionada ao hemisfério nãodominante (Springer \& Deutsch, 1998) ou ao hemisfério direito, em tarefas de reconhecimento de tons (Beaver \& Chiarello, 1974), na escuta dicótica (Kimura, 1964), na estimulação cerebral de regiões específicas (Penfield \& Jasper, 1954) e nos estudos de neuropatologia, tal qual a epilepsia musicogênica (Critchley, 1937).

Esse aspecto aponta possíveis limitações de não se possuírem metodologias específicas para o estudo da cognição musical e, por consequência, do ouvido absoluto, uma vez que o processamento cerebral dos conteúdos que caracterizam o fenômeno musical distingue-se do processamento da linguagem formal. Em estudo recente sobre ouvido absoluto, verificou-se que a nomeação de notas musicais em pessoas com a habilidade, em comparação com pessoas que não a possuem, gera maior atividade neural em ambos os hemisférios cerebrais (Wu, Kirk, Hamm \& Lim, 2008). 
Se, por um lado, isso sugere uma organização do conteúdo tonal equivalente à organização cerebral para a formação da linguagem formal, por outro evidencia participação de estruturas de maneira distinta no processamento musical.

Seria importante, portanto, que o estudo científico do tema incluísse o desenvolvimento de metodologias próprias, a fim de garantir resultados mais robustos e condizentes com os fatores que o permeiam. Embora bem adaptados ao contexto, como os estudos psicofísicos e de imageamento cerebral, a herança de metodologias empregadas na linguagem formal não é incomum. Termos como "gramática musical" e aspectos "léxicos" da música são amplamente utilizados e sugerem uma adaptação do tema a outra área de investigação neurocientífica, ainda que as diferenças proeminentes entre elas sejam pontuadas. Ou seja, a adoção de metodologias mais conhecidas para averiguação de um fenômeno ainda pouco estudado no meio científico é um percurso natural. No entanto, o investimento em delineamento que considere as propriedades inerentes ao fenômeno, pontuando-se os aspectos básicos da estrutura musical, tal qual timbre, altura tonal, contorno melódico, ritmo, por exemplo, é um passo importante para a melhor elucidação da habilidade.

\section{Conclusão}

Uma reflexão meticulosa acerca dos pontos sublinhados leva à conclusão de que as lacunas vinculadas à melhor compreensão do Ouvido Absoluto, tal qual uma definição mais concisa, fatores intrínsecos ao desenvolvimento da habilidade, sua origem, desenvolvimento e implicações para o entendimento acerca da cognição musical tornam-se evidentes.

Em primeiro lugar, destaca-se que a própria definição de ouvido absoluto é controversa. A ausência de delineamentos experimentais com sujeitos que não possuam a capacidade de nomear um tom musical, ou seja, desvinculando o pressuposto de estudo teórico musical formal, mostra-se um ponto fundamental. O ajuste de metodologias que possibilitem o estudo da condição nessa população específica pode trazer ganhos essenciais para a melhor compreensão do fenômeno. A utilização de exames de imagem consegue trazer indícios da ativação cerebral inerente ao ouvido absoluto, embora não sejam suficientes para discorrer a complexidade da habilidade. $O$ que se observa com tais estudos é um mapa funcional ainda em investigações iniciais. No entanto, uma explanação acerca das causas e efeitos de tais modificações, bem como inferências acerca dos aspectos perceptuais e cognitivos inerentes, ainda estão longe de serem alcançadas.
Um segundo aspecto relevante diz respeito às divergências acerca da gênese do ouvido absoluto. Até que ponto os aspectos constitucionais favorecem o aparecimento da habilidade em detrimento dos fatores ambientais como seu determinante? Mesmo que algumas pesquisas proponham um entendimento convergente desses aspectos como favorecedores de sua ocorrência, os estudos ainda são relativamente recentes, sugerindo, tão somente, uma alternativa à explanação do tema. Parece correto afirmar que o ouvido absoluto é uma habilidade que modifica o funcionamento cerebral naqueles que o possuem. A melhor elucidação dos substratos neurais que influenciam a percepção do estímulo auditivo nessa população pode esclarecer questões importantes sobre os mecanismos cerebrais e processos mentais associados à música. Da mesma forma, a compreensão das mudanças funcionais em virtude da influência ambiental e a correlação entre as alterações nos aspectos estruturais cerebrais em decorrência dos fatores constitucionais também são tópicos importantes para a compreensão da atividade cerebral diante de estímulos musicais e possíveis implicações.

Complementarmente, destaca-se que grande parte dos estudos acerca do ouvido absoluto considera tarefas com aspectos inerentes ao objeto musical (timbre, tons, ritmo etc.) isoladamente ou em frases musicais que não correspondem necessariamente ao que comumente caracteriza a música (um fenômeno complexo esculpido no tempo). O desenvolvimento e a combinação de técnicas e metodologias ajustadas ao estudo da música parecem ser condições necessárias para maiores avanços na área.

Por fim, deve-se ressaltar que não se tem clareza acerca das reais vantagens que pessoas com ouvido absoluto possuem, em detrimento das que não o possuem. Efetivamente, os estudos não sugerem, até o momento, uma diferença no que tange ao processamento da informação musical entre os dois grupos e, sim, uma diferença no que se refere ao desempenho de tarefas musicais específicas (Miyazaki \& Rakowski, 2002), como no reconhecimento de tons isolados. Entender a natureza da habilidade e a particularidade do ouvido absoluto a um grupo específico da população pode trazer uma melhor compreensão acerca da percepção de tom, das bases da cognição musical e de quais fatores interferem no processamento desse tipo de informação auditiva.

\section{Referências}

Bachem, A. (1937). Various types of absolute pitch. The Journal of the Acoustical Society of America, 9, 146-157.

Bachem, A. (1955). Absolute pitch. The Journal of the Acoustical Society of America, 27, 1180-1185.

Baharloo, S., Johnston, P. A., Service, S. K., Gitschier, J. \& Freimer, N. B. (1998). Absolute pitch: an approach for identification of genetic and 
nongenetic components. The American Journal of Human Genetics, 62(2), 224-231.

Baird, A. \& Samson, S. (2009). Memory for music in Alzheimer's disease: unforgetable? Neuropsychological Review, 19, 85-101.

Beaver, T. G. \& Chiarello, R. J. (1974). Cerebral dominance in musicians and nonmusicians. Science, 185, 537-539.

Brancucci, A., di Nuzzo, M. \& Tommasi, L. (2009). Opposite hemispheric asymmetries for pitch identification in absolute pitch and non-absolute pitch musicians. Neuropsychologia, 47(13), 2937 2941. doi: 10.1016/j.neuropsychologia.2009.06.021

Creel, S. C. \& Tumlin, M. A. (2012). Online recognition of music is influenced by relative and absolute pitch information. Cognitive Science, 36, 224-260.

Critchley, M. (1937). Musicogenic epilepsy. Brain 60, 1328-1337.

Deutsch, D. (2002). The puzzle of absolute pitch. Current Directions in Psychological Science, 11(6), 200204. DOI: $10.1111 / 1467-8721.00200$.

Deutsch, D., Dooley, K., Henthorn, T. \& Head, B. (2009). Absolute pitch among students in an American music conservatory: association with tone language fluency. The Journal of the Acoustical Society of America, 125(4), 2398.

Gregersen, P., Kowalsky, E., Kohn, N. \& Marvin, E. (2000). Early childhood music education and predisposition to absolute pitch: teasing apart genes and environment. American Journal of Medical Genetics, 98, 280-282.

Halpern, A. R. (1989). Memory for the absolute pitch of familiar songs. Memory \& Cognition, 17(5), 572581.

Kandel, E. R., Schwartz, J. H. \& Jessel, T. M. (1995). Fundamentos da neurociência e do comportamento. São Paulo: Prentice-Hall do Brasil.

Kimura, D. (1964). Left-right differences in perception of melodies. Quarterly Journal of Experimental Psychology, 16, 335-358.

Levitin, D. J. (1994). Absolute memory for musical pitch: evidence from the production of learned memories. Perception \& Psychophysics, 56(4), 414423.

Levitin, D. J. \& Rogers, S. E. (2005). Absolute pitch: perception, coding, and controversies. Trends in Cognitive Sciences, 9(1), 26-33. doi: 10.1016/j.tics.2004.11.007.

Levitin, D. J. \& Tirovolas, A. K. (2009). Current advances in the cognitive neuroscience of music. Annals of the New York Academy of Sciences, 1156(1), 211-231. doi: 10.1111/j.1749-6632.2009.04417.x

Loui, P., Li, H. C., Hohmann, A. \& Schlaug, G. (2011). Enhanced cortical connectivity in absolute pitch musicians: a model for local hyperconnectivity. Journal of Cognitive Neuroscience, 23(4), 1015-1026.
Miyazaki, K. (1988). Musical pitch identification by absolute pitch possessors. Perception \& Psychophysics, 44(6), 501-512.

Miyazaki, K. \& Rakowski, A. (2002). Recognition of notated melodies by possessors and nonpossessors of absolute pitch. Perception \& Psychophysics, 64, 1337-1345.

Oechslin, M. S., Meyer, M. \& Jancke, L. (2009). Absolute pitch: functional evidence of speechrelevant auditory acuity. Cerebral Cortex, 20(2), 447455. doi: 10.1093/cercor/bhp113.

Penfield, J. \& Jasper, H. L. (1954). Epilepsy and the functional anatomy of the brain buman. Boston: Little Brown and Company.

Profita, J. \& Bidder, T. G. (1988). Perfect pitch. American Journal of Human Genetic, 29, 763-771.

Roederer, J. G. (2002). Introdução à física e psicofísica da música. São Paulo: Editora da Universidade de São Paulo.

Ross, D. A., Olson, I. R. \& Gore, J. C. (2003). Absolute pitch does not depend on early musical training. Annals of the New York Academy of Sciences, 999, 522-526.

Ross, D., Gore, J. \& Marks, L. (2005). Absolute pitch: music and beyond. Epilepsy \& Behavior, 7(4), 578601. doi: 10.1016/j.yebeh.2005.05.019.

Ross, D. A., Olson, I. R., Marks, L. E. \& Gore, J. C. (2004). A non-musical paradigm for identifying absolute pitch possessors. The Journal of the Acoustical Society of America, 116, 1793-1799.

Schellenberg, E. G. \& Trehub, S. (2003). Good pitch memory is widespread. Psychological Science, 14(3), 262-266.

Schlaug, G., Jancke, L., Huang, Y. \& Steinmetz, H. (2005). In vivo evidence of structural brain asymmetry in musicians. Science, 267, 699-701.

Schulze, K, Mueller, K. \& Koelsch, S. (2012). Auditory stroop and absolute pitch: an fMRI study. Human Brain Mapping. doi: 10.1002/hbm.22010.

Smith, N. A. \& Schmukler, M. A. (2004). The perception of tonal structure through the differentiation and organization of pitches. Journal of Experimental Psychology: Human Perception and Performance, 30(2), 268-286.

Springer, P. S. \& Deutsch, G. (1998). Cérebro esquerdo, cérebro direito. São Paulo: Summus.

Takeushi, A. H. \& Hulse, S. H. (1993). Absolute pitch. Psychological Bulletin, 113, 345-361.

Terhardt, T. \& Seewann, M. (1983). Aural key identification and its relationship to absolute pitch. Music Perception, 1, 63-83.

Theusch, E. \& Gitschier, J. (2011). Absolute pitch twin study and segregation analysis. Twin Research and Human Genetics, 14(2), 173-178.

Ward, W. D. (1999). Absolute pitch. Em D. Deutsch (Ed.), The Psychology of Music (pp. 265-294). California: Academic Press. 
Wilson, S. J., Lusher, D., Martin, C. L., Rayner, G. \& McLachlan, N. (2012). Intersecting factors lead to absolute pitch acquisition that is maintained in a "fixed do" environment. Music Perception, 29(3), 285-296.

Wilson, S. J., Lusher, D., Wan, Y., Dudgeon, P., Reutens, D. C. (2009). The neurocognitive components of pitch processing: insights from absolute pitch. Cerebral Cortex, 19, 724-732.

Wu, C., Kirk, I. J., Hamm, J. P. \& Lim, V. K. (2008). The neural networks involved in pitch labeling of absolute pitch musicians. Cognitive Neuroscience and Neuropathology, 18(8), 851-854.

Zatorre, R. J. (2003). Absolute pitch: a model for understanding the influences of genes and development on neural and cognitive function. Nature Neuroscience, 6(7), 692-695.
Zatorre, R. J. \& Beckett, C. (1989). Multiple coding strategies in the retention of musical tones by possessors of absolute pitch. Memory \& Cognition, 17(5), 582-589.

Zatorre, R. J., Perry, D. W., Beckett, C. A., Westbury, C. F. \& Evans, A. C. (1998). Functional anatomy of musical processing in listeners with absolute pitch and relative pitch. Proceedings of the National Academy of Sciences of the United States of America, 95(6), 3172-3177.

Recebido em 15/08/2011

Primeira Reformulação em 22/10/2012

Segunda Reformulação em 29/05/2013

Aprovado em 23/10/2013

Sobre os autores:

Fabrízio Veloso é neuropsicólogo e psicólogo, mestre em Processos Psicológicos Básicos pela Universidade de Brasília e doutorando pelo programa de Neurociências e Comportamento da Universidade de São Paulo, área de pesquisa em cognição musical.

Maria Angela Guimarães Feitosa é PhD pela The University of Michigan, Ann Arbor, EUA, professora titular junto ao Depto de Processos Psicológicos Básicos, Instituto de Psicologia, Universidade de Brasília.

Contato com os autores:

Maria Angela Guimarães Feitosa - Departamento de Processos Psicológicos Básicos, Instituto de Psicologia - Universidade de Brasília - Campus Universitário Darcy Ribeiro, Instituto Central de Ciências Sul, CEP 70910-900 - Brasília/DF.

E-mail: feitosa.mag@gmail.com

Fabrízio Veloso - Instituto de Ciências Biomédicas - Universidade de São Paulo - Departamento de Fisiologia e Biofísica, Avenida Prof. Lineu Prestes 1524, Cidade Universitária, CEP 05508-900 - São Paulo, SP

E-mail: fabrizioveloso@gmail.com 